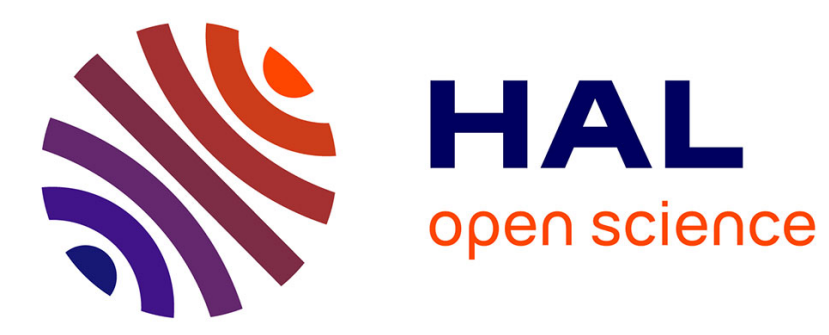

\title{
Role of Transient Reflection in Graphene Nonlinear Infrared Optics
}

Ryan J. Suess, Stephan Winnerl, Harald Schneider, Manfred Helm, Claire Berger, Walter A. de Heer, Thomas E. Murphy, Martin Mittendorff

\section{- To cite this version:}

Ryan J. Suess, Stephan Winnerl, Harald Schneider, Manfred Helm, Claire Berger, et al.. Role of Transient Reflection in Graphene Nonlinear Infrared Optics. ACS photonics, 2016, 3 (6), pp.1069 1075. 10.1021/acsphotonics.6b00141 . hal-01784089

\section{HAL Id: hal-01784089 \\ https://hal.science/hal-01784089}

Submitted on 3 May 2018

HAL is a multi-disciplinary open access archive for the deposit and dissemination of scientific research documents, whether they are published or not. The documents may come from teaching and research institutions in France or abroad, or from public or private research centers.
L'archive ouverte pluridisciplinaire HAL, est destinée au dépôt et à la diffusion de documents scientifiques de niveau recherche, publiés ou non, émanant des établissements d'enseignement et de recherche français ou étrangers, des laboratoires publics ou privés. 


\title{
Role of Transient Reflection in Graphene Nonlinear Infrared Optics
}

\author{
Ryan J. Suess, ${ }^{1,}$ a Stephan Winnerl, ${ }^{2}$ Harald Schneider, ${ }^{2}$ Manfred Helm, ${ }^{2,3}$ Claire \\ Berger, ${ }^{4}$ Walter A. de Heer, ${ }^{4}$ Thomas E. Murphy, ${ }^{1}$ and Martin Mittendorff ${ }^{1,2}$ \\ ${ }^{1}$ Institute for Research in Electronics 83 Applied Physics, \\ University of Maryland, College Park, MD 20742, USA \\ ${ }^{2}$ Helmholtz-Zentrum Dresden-Rossendorf, \\ PO Box 510119, D-01314 Dresden, Germany \\ ${ }^{3}$ Technische Universität Dresden, D-01062 Dresden, Germany \\ ${ }^{4}$ Georgia Institute of Technology, Atlanta, GA 30332, USA
}

\begin{abstract}
Understanding the optical response of graphene at terahertz frequencies is of critical importance for designing graphene-based devices that operate in this frequency range. Here we present a terahertz pump-probe measurement that simultaneously measures both the transmitted and reflected probe radiation from multilayer epitaxial graphene, allowing for an unambiguous determination of the pump-induced absorption change in the graphene layers. The photon energy in the experiment $(30 \mathrm{meV})$ is on the order of the doping level in the graphene which enables the exploration of the transition from interband to intraband processes, depending on the amount of pump-induced heating. Our findings establish the presence of a large, photoinduced reflection that contributes to the change in sign of the relative transmitted terahertz radiation, which can be purely positive or predominantly negative depending on the pump fluence, while the change in absorption is found negative at all fluences. We develop a straightforward theory that confirms the sign reversible nature of the relative transmitted terahertz radiation through the graphene multilayer and determine that this behavior originates from either an absorption-bleached or reflection-dominated regime. The theoretical results are incorporated into a model utilizing an energy balance equation that reproduces the measured pump-probe data. These findings, which extend to mid- and far infrared frequencies, illuminate the importance of considering reflection in graphene-light interactions and have implications for the design of future terahertz photonic components.
\end{abstract}

a ryan.suess@gmail.com 


\section{POPULAR SUMMARY}

Despite being technologically underdeveloped compared to optical and radio frequency domains, there remains substantial interest in utilizing terahertz frequencies for applications ranging from astronomy and security to communications systems. Though there has been progress through the development of terahertz emitters and detectors, this range still lacks materials that allow for fabrication of efficient optoelectronic devices that can sense, control, and modify terahertz radiation. Owing to its unique electrical properties and a strong interaction with light, graphene exhibits great potential for the future development of such devices. A thorough understanding of the underlying light-matter interaction in this material is therefore of critical importance for building the next generation of devices that operate at terahertz frequencies. In this work we present a comprehensive experimental study complemented by theoretical work that explores the interaction of high intensity terahertz light with graphene.

The optical properties of graphene are investigated with photons having energies comparable to the chemical potential using terahertz pump-probe spectroscopy. Probing the graphene at intermediate photon energies enables the investigation of the nonlinear optical properties in the graphene as its electron system is heated by the intense pump pulse. By simultaneously measuring the reflected and transmitted terahertz light, a precise determination of the pump-induced change in absorption can be made. We observe that as the intensity of the terahertz radiation is increased, the optical properties of the graphene change from interband, semiconductor-like absorption, to a more metallic behavior with increased intraband processes. This transition reveals itself in our measurements as an increase in the terahertz transmission through the graphene at low fluence, followed by a decrease in transmission and the onset of a large, photoinduced reflection as fluence is increased. Our study further demonstrates the important role that reflection plays in the mid- and far-infrared range - noting that the absorption saturation intensity can be underestimated if only the transmitted light is considered. These findings will contribute to the development of new optoelectronic devices designed to operate in the mid- and far-infrared frequency range. 


\section{INTRODUCTION}

Graphene has attracted tremendous attention as a material suitable for a variety of applications due to its linear and gapless band structure and high carrier mobility. These properties make graphene particularly suitable for applications in the terahertz region, where there are notoriously few materials that interact strongly with electromagnetic radiation of this frequency [1-3]. Significant attention has been given to developing graphene-based terahertz photonic components including devices such as saturable absorbers which are often employed as materials for passive mode-locking [4, 5], terahertz filters $[6,7]$, and broadband detectors $[8,9]$. A detailed understanding of the terahertz-graphene interaction is required to take full advantage of the material, and there have accordingly been many recent studies investigating the heating and cooling characteristics of graphene in the terahertz region[1015], though many have been carried out using a transmission geometry exclusively. Taking into account the pump induced change in reflectivity can reveal new information; for example, the first clear experimental demonstration of carrier multiplication in graphene utilized both reflected and transmitted light[16]. Studies involving terahertz time-domain transmission spectroscopy enable one to extract the complex dynamic conductivity[17-21] but often employ photon energies of a few $\mathrm{meV}$, and are therefore limited to probing intraband absorption only. In this work, we explore a regime of intermediate photon energies, where interband processes also contribute to the optical properties. Here we present a far-infrared pump-probe study that measures both the transmitted and reflected light that enables a comprehensive accounting of the radiation. The data from this experiment reveals the presence of a prominent nonlinear reflection, that saturable absorption is present at all fluences, and a transmission that changes sign with fluence. By employing straightforward theory to determine the graphene optical properties, we show that it is possible, at sufficiently strong intensity, to switch from a primarily interband absorption dominated regime, to one exhibiting significant intraband processes. Together, the measurement and theory give a full picture of the interaction of intense terahertz radiation with low-doped multilayer graphene and provides insight for the design of photonic components that operate in this frequency range. This effect is general in nature and will persist at other wavelengths since it depends primarily on the chemical potential relative to the photon energy. 


\section{MEASUREMENTS}

In this work we investigate multilayer epitaxial graphene (MEG) produced by thermal decomposition of the carbon terminated face of (0001)-oriented silicon carbide. This growth process yields individual layers that are rotated with respect to one another, thus producing multiple decoupled graphene layers[22]. The graphene properties were verified using angular resolved photoemission spectroscopy to ensure linear dispersion[23] while the thickness was measured using ellipsometry, which indicated that the sample consists of approximately 50 layers. Investigation using magneto-spectroscopic measurements reveal long momentum relaxation times that can be attributed to low defect densities (scattering time: $\tau_{s}=300 \mathrm{fs}$ )[24] and extremely low doping for the majority of the layers (in the range of 8-15 meV)[24, 25]. It should be noted that the layers near the SiC-graphene interface can have larger doping levels $(\approx 300 \mathrm{meV})$ due to interfacial charge redistribution from the SiC substrate. The doping levels decrease rapidly within the first few layers away from the substrate, leaving a mostly intrinsic graphene multilayer [25-27].

To carry out a pump-probe study in which both reflected and transmitted light are collected, an off-axis parabolic mirror (OAP) is used (cf. Fig. 1). This approach allows spatial separation of the pump and probe pulses in both the transmitted and reflected directions and produces a focussed spot size of $500 \mu \mathrm{m}$. The beam path begins with high fluence pulses generated from a free-electron laser (FEL)[28] being directed through a beam splitter which separates the beam in to pump and probe paths. The pulses from the FEL have a center wavelength of $42 \mu \mathrm{m}(30 \mathrm{meV})$ or equivalently a center frequency of $\approx 7 \mathrm{THz}$, pulsewidth of 7.5 ps FWHM, and a repetition rate of $13 \mathrm{MHz}$. The pump and probe beam paths are independently attenuated and are linearly cross-polarized. The pump was chopped using a mechanical chopper and a pump-to-probe power ratio of around 25 was maintained for all measurements reported in the study. As shown in Fig.1, the probe beam is delayed with respect to the pump pulse using a delay stage that allows for delays of up to 250 ps to be scanned.

Pump-probe measurements are carried out on a MEG sample for a variety of pump fluences spanning over two orders in magnitude $\left(25 \mathrm{~nJ} \cdot \mathrm{cm}^{-2}\right.$ to $\left.8.6 \mu \mathrm{J} \cdot \mathrm{cm}^{-2}\right)$ at a temperature of $10 \mathrm{~K}$. The pump-induced modulation on the reflected and transmitted probe pulse is measured using lock-in detection; producing the values $\Delta \mathcal{T}$ and $\Delta \mathcal{R}$ respectively. The 
changes in transmission and reflection can be specified as a percentage by dividing $\Delta \mathcal{T}$ and $\Delta \mathcal{R}$ by the signal obtained by chopping the probe directly and measuring the transmitted and reflected signals when no pump is present. This gives, respectively, the relative change in transmission and reflection $\Delta \mathcal{T} / \mathcal{T}$ and $\Delta \mathcal{R} / \mathcal{R}$. The pump-induced change in absorption is calculated from the transmission and reflection measurements using the relation $\Delta \mathcal{A}=-(\Delta \mathcal{T}+\Delta \mathcal{R})$. Since an accurate measurement of the absorbed probe is difficult, $\Delta \mathcal{A}$ cannot be easily normalized to a relative value, as was done for the transmitted and reflected signals, and is therefore given in arbitrary units.

Figure 2 shows the measured change in transmission, reflection, and absorption as a function of probe delay and pump fluence. In Fig.2(a) the relative change in tranmission exhibits a fluence dependent sign change apparent as red and blue regions in the colormap. At pump fluences below $0.6 \mu \mathrm{J} \cdot \mathrm{cm}^{-2}$ the transmitted signal is positive suggesting that saturable absorption is dominant in the terahertz transmission for low pump fluences. When the pump fluence increases beyond $3 \mu \mathrm{J} \cdot \mathrm{cm}^{-2}$ the sign of the terahertz transmission becomes predominantly negative; indicating either a pump-induced absorption effect or reflection is responsible for the decrease in transmission. The relative change in reflection is displayed in Fig. 2(b) and exhibits a strictly positive signal that increases monotonically with pump fluence. The positive nature of this signal strongly indicates that the negative relative transmission at high pump fluences is at least partially caused by an increase in the graphene reflectivity. A similar monotonic trend is seen for the change in absorption, (cf. Fig. 2(c)) which is entirely negative, verifying that the MEG behaves as a saturable absorber over the entire range of pump fluences investigated. As a result of the strong pump-induced reflection, determination of the absorption saturation fluence using only the transmission data can lead to a significant underestimation of its actual value. In the present work, the absorption saturation fluence was estimated[29] from the positive portion of the transmission data to be $8 \mathrm{~mW}$, seven times smaller than the value determined by including both transmitted and reflected light in the estimation $(56 \mathrm{~mW})$. An accurate accounting of the saturation fluence and the presence of a large, photoinduced reflection are important considerations for graphene-based saturable absorbers employed in laser mode-locking applications[30]. This also creates the possibility of using graphene as a broadband saturable absorbing mirror, a device that traditionally relies on semiconductor materials to achieve an enhanced reflectivity as pulse fluence is increased[31]. 


\section{THEORY}

To understand the transient changes in the optical properties, we first consider the temperature dependent conductivity of graphene. We assume that thermalized distribution is achieved in the material during the pump excitation which is well justified by noting that the carrier-carrier scattering processes through which the electron population is heated is much faster than the temporal resolution of our experiment. We can calculate the temperature dependent terahertz conductivity using[32]:

$$
\sigma(\omega, T)=\frac{e^{2}(\hbar \omega)}{i \pi \hbar}\left(\int_{-\infty}^{+\infty} d E \frac{|E|}{(\hbar \omega)^{2}} \frac{\partial f(E, T)}{\partial E}-\int_{0}^{+\infty} d E \frac{f(-E, T)-f(E, T)}{(\hbar \omega)^{2}-4 E^{2}}\right) .
$$

In the above expression $E$ is the energy, $e$ is the electron charge, $\omega$ is the frequency of the illumination, $\hbar$ is the reduced Planck's constant, and $f(E, T)=\left(\exp \left[(E-\mu(T)) / k_{B} T\right]+1\right)^{-1}$ is the Fermi function, where $\mu(T)$ is the temperature dependent chemical potential, $k_{B}$ is the Boltzmann constant, and $T$ is the electron temperature. The first and second terms in Eqn. 1 describe the intraband and interband conductivity respectively. The intraband portion can be evaluated to give: $\frac{2 i e^{2}}{\pi \hbar} \frac{k_{B} T}{\hbar\left(\omega+i \tau_{s}^{-1}\right)} \ln \left[\frac{2 \cosh (\mu(T)}{2 k_{B} T}\right]$, where $\tau_{s}$ is the electron scattering time, while the interband portion is evaluated numerically[32]. The number of charge carriers is conserved in our calculations $\left(n=\int_{-\infty}^{+\infty} D(E) f(E) d E\right.$ where $D(E)$ is the graphene density of states), and defines an implicit relationship between the chemical potential and temperature[32, 33]. In the following analysis, we attribute the optical response of the MEG to be entirely from the low-doped layers[11] which comprise the majority of our multilayer graphene sample.

The media surrounding the graphene impacts the optical properties expected from the combined superstrate-graphene-substrate interface[34]. The temperature dependent conductivity is calculated and then used to find the temperature dependent optical parameters $(\Delta \mathcal{T} / \mathcal{T}, \Delta \mathcal{R} / \mathcal{R}$, and $\Delta \mathcal{A} / \mathcal{A})$ for the well known case of a lossy film of thickness, $d$, separating two materials with permittivities $\epsilon_{a}$ and $\epsilon_{b}[35]$. In this calculation we use a thickness corresponding to 50 graphene layers and a super-/substrate permittivity of 1 (vacuum)/12.3 (SiC). Charge impurities in the substrate material as well as surface topography and defects can further influence the properties of the graphene through the formation of spatial charge puddle inhomogeneities which modify the transport properties in low-doped graphene[3639]. In the present work, the far-infrared beam samples a large area of the multilayer 
graphene containing a spatially inhomogeneous distribution of chemical potential patches (length scale for potential patches have been reported to be of order $10^{2} \mathrm{~nm}[40]$ ). The beam averages over these patches and effectively samples a broadened Fermi distribution edge. The puddle potential distribution has been measured experimentally[38, 40] and considered theoretically using Gaussian statistics[36]. Optical parameters consistent with the experimental data are produced by convolving the Fermi distributions used in our calculations with a charge puddle distribution having a standard deviation (fluctuation strength) of approximately $20 \mathrm{meV}[36]$.

Figure 3 shows the change in optical coefficients as a function of change in temperature at a lattice temperature of $\mathrm{T}_{\ell}=10 \mathrm{~K}$. The reflection and absorption curves are seen to be entirely monotonically increasing and decreasing functions of change in temperature respectively, while the relative change in transmission changes sign, becoming negative for temperature changes higher than $140 \mathrm{~K}$. The absorption and reflection curves illustrate that this behavior emerges when the influence of the pump-induced reflectivity surpasses that of the interband absorption bleaching. This bleaching appears as a negative change in the absorption in Fig. 3 and is the result of Pauli-blocking. This effect is dominant over the pump-induced reflection for small changes in temperature and leads to a net positive $\Delta \mathcal{T} / \mathcal{T}$. It will appear at other frequencies in the infrared where the doping and photon energy are of similar scale and changes in temperature from the absorbed radiation will sufficiently broaden the Fermi distribution.

Having established the role of the pump-induced reflection in the optical transmission in graphene, we next demonstrate that the derived temperature dependent optical parameters will lead directly to pump-probe signals with identical characteristics to those measured experimentally. An energy balance equation is used to form a relationship between the incident power and the pump-induced change in temperature in the graphene. For the sake of clarity, a model consisting of only the essential heating and cooling terms, similar to those used by others studying monolayer graphene[41-43], are used to describe the thermodynamics of the multilayer graphene in our pump-probe experiment. The resulting nonlinear differential equation contains terms for the electronic heat capacity, cooling, and a self-consistent absorption/heating term:

$$
\mathcal{C}_{e l}(T) \frac{d T}{d t}=\mathcal{A}(T) \eta P(t)-g_{1}\left(T-T_{l}\right)
$$


The terms in the energy balance equation are given as follows: $\mathcal{C}_{e l}$ denotes the electronic specific heat capacity, where $\mathcal{C}_{e l}(T)=\left(\frac{18 \zeta(3)}{\pi \hbar^{2} v_{F}^{2}}\right) T^{2}$ for our low-doped graphene [44-46] where $v_{F}$ denotes the Fermi velocity and $\zeta$ is the Riemann zeta function. The term $\mathcal{A}(T)$ is the temperature dependent absorption as determined from our previous analysis of a lossy film separating two dielectrics. A phenomenological heating efficiency term[33], $\eta \approx 0.03$, was introduced into Eqn. 2 to achieve similar fluence scaling between the thermodynamic model and the data by scaling the incident power. The efficiency factor accounts for all of the unknown parameters in the energy balance equation with a single term. The incident laser pulse heating the graphene is described by a Gaussian envelope, $P(t)$. The parameter $g_{1}=T^{4} \frac{7 \pi^{3} k_{B}^{5} D^{2}}{30 \hbar^{5} v_{F}^{6}}$ is the cooling power coefficient in the clean limit when the temperature is above the Bloch-Gruneisen temperature[43] ( $T_{B G}<10 \mathrm{~K}$ for the low-doped layers), $D \approx 2 \mathrm{eV}$ is the deformation potential, $v_{s}=2 \times 10^{4} \mathrm{~m} / \mathrm{s}$ is the sound velocity in graphene, and $\rho=7.6 \times 10^{-7} \mathrm{~kg} / \mathrm{m}^{2}$ is the mass density of graphene. Equation 2 is solved self-consistently to give the change in temperature as a function of time and used to calculate the delaydependent optical parameters in the weak probe limit (i.e., the temperature change results solely from the pump pulse). The left panel of Fig. 4 shows the optical parameters produced by the measurement at selected pump fluences defined in Fig. 1 while the right panel of Fig. 4 shows the corresponding curves determined by the model.

\section{DISCUSSION}

As can be seen from Fig. 4(a), the model and data show relative transmission curves that are strictly positive at low fluence $\left(<0.6 \mu \mathrm{J} \cdot \mathrm{cm}^{-2}\right)$ and mostly negative at higher fluences $\left(>3 \mu \mathrm{J} \cdot \mathrm{cm}^{-2}\right)$. The relative change in reflection from the model and data are presented in Fig. 4(b). These curves display a relative reflection that rapidly increases with pump fluence. The change in absorption curves decrease with pump fluence as seen in Fig. 4(c). The transmission curves change sign as a function of fluence when the increasing reflection becomes larger than the steadily decreasing absorption; marking a change from a fluence regime exhibiting primarily saturable absorption, to one exhibiting primarly pump-induced reflection. This changeover in phenomenology takes place when heating reduces the amount of interband absorption due to a decreased difference in carrier occupation at $\pm \hbar \omega / 2$ and a simultaneous increase in intraband conductivity, which scales with temperature, that pro- 
duces a change in reflectivity. Figure 5(a) plots the Fermi function for a range of temperature changes and shows the degree of the temperature dependent change in chemical potential and edge broadening with respect to the incident photon energy. The effect of disorder can be observed as a broadened width of the Fermi function at low temperatures.

The real and imaginary components of the graphene conductivity as determined by Eqn. 1 are plotted in Fig. 5 (b) and (c) respectively. Here the conductivity resulting from interband and intraband processes are plotted independently (dash-dotted and dashed lines respectively) and together add up to the total conductivity (solid trace). As shown in the figure, the heating modifies the conductivity of the graphene and results in inter- and intraband processes playing a diminished and increased role respectively (an estimated peak change in temperature of around $200 \mathrm{~K}$ was reached in our experiments). Furthermore, since absorption in the graphene is proportional to the real part of the conductivity, the decrease shown in Fig. 5(b) confirms that the graphene behaves as a saturable absorber for all fluences in our experiment and that the sign change in the transmitted signal results exclusively from an increase in the reflectivity.

The observed sign change in transmission was previously shown to originate from a temperature dependent change in inter- and intraband conductivity. To generalize this result, we consider the temperature at which the real component of the conductivity from interband and intraband processes are equal for a range of photon energies and carrier concentrations (plotted in Fig. 6 as solid colored lines). Though the temperature at which the conductivity contributions from interband and intraband processes achieve parity was not reached in our experiment, this temperature identifies a general point of comparison for the optical properties studied in this work. Analogous energy scales to the ones used in our experiment are represented for the case where the incident photon energy is in the vicinity of the chemical potential (shown as dashed colored lines in Fig. 6) where, with increasing temperature, the conductivity transitions from being interband to intraband dominant as in Fig. 5(b). For photon energies well below the chemical potential, the crossover temperature represents the crossover from intraband to interband processes. Above the chemical potential, all curves asymptotically approach the high temperature limit $\left(k_{B} T \gg \mu(T)\right.$, black dashed line) which is independent of the graphene doping. In the high temperature limit, the crossover temperature increases with photon energy which means that at higher photon energies, the electrons must be heated by a greater amount in order for the intraband processes to compete with 
the interband ones. At low temperature and doping, the disorder-broadened Fermi function limits the solution to finite valued photon energies. The conductivity crossover temperatures in Fig. 6 are well within the range of experimentally observed values[11, 20,33] and indicate that the same phenomena will be observed for a range of wavelengths spanning the midand far-infrared frequency range.

\section{CONCLUSION}

We have investigated the terahertz response of low-doped multilayer epitaxial graphene using pump-probe spectroscopy that measures both the reflected and transmitted farinfrared radiation. This radiometrically comprehensive measurement allows for clear determination of the absorption in the material which was shown to be negative, and monotonic with fluence; verifying that the graphene behaves as a saturable absorber. Additionally, the relative change in the terahertz transmission was observed to change sign depending on the magnitude of the pump fluence as the result of a large, photoinduced reflection. This

effect was clearly explained by a straightforward theory that identified two distinct physical mechanisms for the terahertz optical properties: one at low fluence where the properties are dominated by interband absorption of the incident terahertz photons, and a metallic regime that is characterized by increased intraband processes resulting from a heated electron system with significant carrier populations above the incident photon energy. These results demonstrate that it is possible to observe a self-induced modification of the absorption mechanism and underscores the importance of considering reflection graphene optics. These findings apply to a range of mid- and far-infrared frequencies and will be important for the design of next generation graphene-based terahertz devices.

\section{ACKNOWLEDGEMENTS}

This work was sponsored by the US ONR (N000141310865) and the US NSF (ECCS 1309750). We are grateful to P. Michel and the FELBE team for their dedicated support. 


\section{REFERENCES}

[1] T. Otsuji, S.A. Boubanga Tombet, A. Satou, H. Fukidome, M. Suemitsu, E. Sano, V. Popov, M. Ryzhii, and V. Ryzhii, "Graphene-based devices in terahertz science and technology," J. Phys. D: Appl. Phys. 45, 303001 (2012).

[2] F. Bonaccorso, Z. Sun, T. Hasan, and A.C. Ferrari, "Graphene photonics and optoelectronics," Nature Photon. 4, 611-622 (2010).

[3] A. Tredicucci and M.S. Vitiello, "Device concepts for graphene-based terahertz photonics," IEEE J. Sel. Top. Quantum Electron. 20, 130-138 (2014).

[4] Z. Sun, T. Hasan, F. Torrisi, D. Popa, G. Privitera, F. Wang, F. Bonaccorso, D.M. Basko, and A.C. Ferrari, "Graphene mode-locked ultrafast laser," ACS Nano 4, 803-810 (2010).

[5] Q. Bao, H. Zhang, Y. Wang, Z. Ni, Y. Yan, Z.X. Shen, K.P. Loh, and D.Y. Tang, "Atomiclayer graphene as a saturable absorber for ultrafast pulsed lasers," Adv. Funct. Mater. 19, 3077-3083 (2009).

[6] M.M. Jadidi, A.B. Sushkov, R.L. Myers-Ward, A.K. Boyd, K.M. Daniels, D.K. Gaskill, M.S. Fuhrer, H.D. Drew, and T.E. Murphy, "Tunable terahertz hybrid metal-graphene plasmons," Nano Lett. 15, 7099-7104 (2015).

[7] L. Ju, B. Geng, J. Horng, C. Girit, M. Martin, Z. Hao, H.A. Bechtel, X. Liang, A. Zettl, Y-.R. Shen, and F. Wang, "Graphene plasmonics for tunable terahertz metamaterials," Nat. Nanotechnol. 6, 630-634 (2011).

[8] M. Mittendorff, J. Kamann, J. Eroms, D. Weiss, C. Drexler, S.D. Ganichev, J. Kerbusch, A. Erbe, R.J. Suess, T.E. Murphy, S. Chatterjee, K. Kolata, J. Ohser, J.C. König-Otto, H. Schneider, M. Helm, and S. Winnerl, "Universal ultrafast detector for short optical pulses based on graphene," Opt. Express 23, 28728-28735 (2015).

[9] F. Bianco, D. Perenzoni, D. Convertino, S. L. De Bonis, D. Spirito, M. Perenzoni, C. Coletti, M. S. Vitiello, and A. Tredicucci, "Terahertz detection by epitaxial-graphene field-effecttransistors on silicon carbide," Appl. Phys. Lett. 107, 131104 (2015).

[10] J.M. Dawlaty, S. Shivaraman, M. Chandrashekhar, F. Rana, and M.G. Spencer, "Measurement of ultrafast carrier dynamics in epitaxial graphene," Appl. Phys. Lett. 92, 042116 (2008).

[11] P.A. George, J. Strait, J. Dawlaty, S. Shivaraman, M. Chandrashekhar, F. Rana, and M.G. Spencer, "Ultrafast optical-pump terahertz-probe spectroscopy of the carrier relaxation and 
recombination dynamics in epitaxial graphene," Nano Lett. 8, 4248-4251 (2008).

[12] H. Choi, F. Borondics, D. A. Siegel, S. Y. Zhou, M. C. Martin, A. Lanzara, and R. A. Kaindl, "Broadband electromagnetic response and ultrafast dynamics of few-layer epitaxial graphene," Appl. Phys. Lett. 94, 172102 (2009).

[13] K. J. Tielrooij, J. C. W. Song, S. A. Jensen, A. Centeno, A. Pesquera, A. Zurutuza Elorza, M. Bonn, L. S. Levitov, and F. H. L. Koppens, "Photoexcitation cascade and multiple hotcarrier generation in graphene," Nat. Phys. 9, 248-252 (2013).

[14] Z. Mics, K.J. Tielrooij, K. Parvez, S.A. Jensen, I. Ivanov, X. Feng, K. Müllen, M. Bonn, and D. Turchinovich, "Thermodynamic picture of ultrafast charge transport in graphene," Nat. Commun. 6, 7655 (2015).

[15] S. Winnerl, M. Orlita, P. Plochocka, P. Kossacki, M. Potemski, T. Winzer, E. Malic, A. Knorr, M. Sprinkle, C. Berger, W. A. de Heer, H. Schneider, and M. Helm, "Carrier Relaxation in Epitaxial Graphene Photoexcited Near the Dirac Point," Phys. Rev. Lett. 107, 237401 (2011).

[16] T. Plötzing, T. Winzer, E. Malic, D. Neumaier, A. Knorr, and H. Kurz, "Experimental verification of carrier multiplication in graphene," Nano Lett. 14, 5371-5375 (2014).

[17] J. Horng, C.F. Chen, B. Geng, C. Girit, Y. Zhang, Z. Hao, H.A. Bechtel, M. Martin, A. Zettl, M.F. Crommie, Y-.R. Shen, and F. Wang, "Drude conductivity of dirac fermions in graphene," Phys. Rev. B 83, 165113 (2011).

[18] A. J. Frenzel, C. H. Lui, Y. C. Shin, J. Kong, and N. Gedik, "Semiconducting-to-Metallic Photoconductivity Crossover and Temperature-Dependent Drude Weight in Graphene," Phys. Rev. Lett. 113, 056602 (2014).

[19] H.Y. Hwang, N.C. Brandt, H. Farhat, A.L. Hsu, J. Kong, and K.A. Nelson, "Nonlinear THz Conductivity Dynamics in P-Type CVD-Grown Graphene,” J. Phys. Chem. B 117, 1581915824 (2013).

[20] G. Jnawali, Y. Rao, H. Yan, and T.F. Heinz, "Observation of a Transient Decrease in Terahertz Conductivity of Single-Layer Graphene Induced by Ultrafast Optical Excitation," Nano Lett. 13, 524-530 (2013).

[21] H.A. Hafez, P.L. Lévesque, I. Al-Naib, M.M. Dignam, X. Chai, S. Choubak, P. Desjardins, R. Martel, and T. Ozaki, "Intense terahertz field effects on photoexcited carrier dynamics in gated graphene," Appl. Phys. Lett. 107, 251903 (2015). 
[22] J. Hass, F. Varchon, J. E. Millán-Otoya, M. Sprinkle, N. Sharma, W. A. de Heer, C. Berger, P. N. First, L. Magaud, and E. H. Conrad, "Why multilayer graphene on $4 h-\operatorname{SiC}(000 \overline{1})$ behaves like a single sheet of graphene," Phys. Rev. Lett. 100, 125504 (2008).

[23] M. Sprinkle, D. Siegel, Y. Hu, J. Hicks, A. Tejeda, A. Taleb-Ibrahimi, P. Le Fèvre, F. Bertran, S. Vizzini, H. Enriquez, S. Chiang, P. Soukiassian, C. Berger, W. A. de Heer, A. Lanzara, and E. H. Conrad, "First direct observation of a nearly ideal graphene band structure," Phys. Rev. Lett. 103, 226803 (2009).

[24] M. Orlita, C. Faugeras, P. Plochocka, P. Neugebauer, G. Martinez, D. K. Maude, A.-L. Barra, M. Sprinkle, C. Berger, W. A. de Heer, and M. Potemski, "Approaching the dirac point in high-mobility multilayer epitaxial graphene," Phys. Rev. Lett. 101, 267601 (2008).

[25] S. Winnerl, F. Göttfert, M. Mittendorff, H. Schneider, M. Helm, T. Winzer, E. Malic, A. Knorr, M. Orlita, M. Potemski, M. Sprinkle, C. Berger, and W. A. de Heer, "Time-resolved spectroscopy on epitaxial graphene in the infrared spectral range: relaxation dynamics and saturation behavior," J. Phys. Condens. Matter 25, 054202 (2013).

[26] D. Sun, Z.-K. Wu, C. Divin, X. Li, C. Berger, W.A. de Heer, P.N. First, and T.B. Norris, "Ultrafast relaxation of excited dirac fermions in epitaxial graphene using optical differential transmission spectroscopy," Phys. Rev. Lett. 101, 157402 (2008).

[27] D. Sun, C. Divin, C. Berger, W.A. de Heer, P.N. First, and T.B. Norris, "Spectroscopic measurement of interlayer screening in multilayer epitaxial graphene," Phys. Rev. Lett. 104, $136802(2010)$.

[28] P. Michel, F. Gabriel, E. Grosse, P. Evtuschenko, T. Dekorsy, M. Krenz, M. Helm, U. Lehnert, W. Seidel, R. Wünsch, D. Wolhlfarth, and A. Wolf, "First lasing of the elbe mid-ir fel," in Proceedings of the 26th FEL Conference, edited by R. Bakker, L. Giannessi, M. Marsi, and R. Walker (Comitato Conferenze Elettra, 2004) pp. 8-13.

[29] E. Garmire, "Resonant optical nonlinearities in semiconductors," IEEE J. Sel. Top. Quantum Electron. 6, 1094-1110 (2000).

[30] N. Tolstik, E. Sorokin, and I.T. Sorokina, "Graphene mode-locked cr:zns laser with 41 fs pulse duration," Opt. Express 22, 5564-5571 (2014).

[31] U. Keller, K.J. Weingarten, F.X. Kärtner, D. Kopf, B. Braun, I.D. Jung, R. Fluck, C. Hönninger, N. Matuschek, and J. Aus der Au, "Semiconductor saturable absorber mirrors (sesam's) for femtosecond to nanosecond pulse generation in solid-state lasers," IEEE J. Sel. 
Top. Quantum Electron. 2, 435-453 (1996).

[32] L. A. Falkovsky and S. S. Pershoguba, "Optical far-infrared properties of a graphene monolayer and multilayer," Phys. Rev. B 76, 153410 (2007).

[33] S. A. Jensen, Z. Mics, I. Ivanov, H. S. Varol, D. Turchinovich, F. H. L. Koppens, M. Bonn, and K. J. Tielrooij, "Competing ultrafast energy relaxation pathways in photoexcited graphene," Nano Lett. 14, 5839-5845 (2014).

[34] J. Holovský, S. Nicolay, S. De Wolf, and C. Ballif, "Effect of the thin-film limit on the measurable optical properties of graphene," Sci. Rep. 5, 15684 (2015).

[35] M. Born and E. Wolf, Principles of Optics, 7th ed. (Cambridge University Press, 1999).

[36] Q. Li, E. H. Hwang, and S. Das Sarma, "Disorder-induced temperature-dependent transport in graphene: Puddles, impurities, activation, and diffusion," Phys. Rev. B 84, 115442 (2011).

[37] E. H. Hwang, S. Adam, and S. Das Sarma, "Carrier transport in two-dimensional graphene layers," Phys. Rev. Lett. 98, 186806 (2007).

[38] Y. Zhang, V.W. Brar, C. Girit, A. Zettl, and M.F. Crommie, "Origin of spatial charge inhomogeneity in graphene," Nat. Phys. 5, 722-726 (2009).

[39] D. Waldmann, J. Jobst, F. Speck, T. Seyller, M. Krieger, and H.B. Weber, "Bottom-gated epitaxial graphene," Nat. Mater. 10, 357-360 (2011).

[40] J. Martin, N. Akerman, G. Ulbricht, T. Lohmann, J.H. Smet, K. Von Klitzing, and A. Yacoby, "Observation of electron-hole puddles in graphene using a scanning single-electron transistor," Nat. Phys. 4, 144-148 (2008).

[41] M.W. Graham, S.-F. Shi, D.C. Ralph, J. Park, and P.L. McEuen, "Photocurrent measurements of supercollision cooling in graphene," Nat. Phys. 9, 103-108 (2013).

[42] A.C. Betz, S.H. Jhang, E. Pallecchi, R. Ferreira, G. Fève, J.-M. Berroir, and B. Plaçais, "Supercollision cooling in undoped graphene," Nat. Phys. 9, 109-112 (2013).

[43] C.B. McKitterick, M.J. Rooks, and D.E. Prober, "Electron-phonon cooling in large monolayer graphene devices," arXiv preprint arXiv:1505.07034 (2015).

[44] C.H. Lui, K.F. Mak, J. Shan, and T.F. Heinz, "Ultrafast photoluminescence from graphene," Phys. Rev. Lett. 105, 127404 (2010).

[45] S.-F. Shi, T.-T. Tang, B. Zeng, L. Ju, Q. Zhou, A. Zettl, and F. Wang, "Controlling graphene ultrafast hot carrier response from metal-like to semiconductor-like by electrostatic gating," Nano Lett. 14, 1578-1582 (2014). 
[46] M.T. Mihnev, J.R. Tolsma, C.J. Divin, D. Sun, R. Asgari, M. Polini, C. Berger, W.A. de Heer, A.H. MacDonald, and T.B. Norris, "Electronic cooling via interlayer Coulomb coupling in multilayer epitaxial graphene," Nat. Commun. 6, 8105 (2015). 
FIGURES 


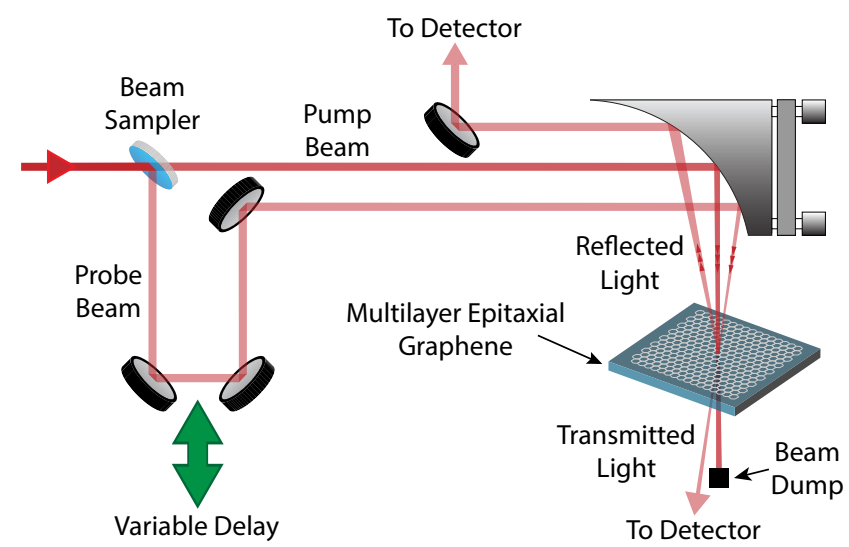

FIG. 1. Diagram showing beam paths for pump and probe beams. The transmitted and reflected portions of the probe beam were measured as a function of the pump-probe delay. 

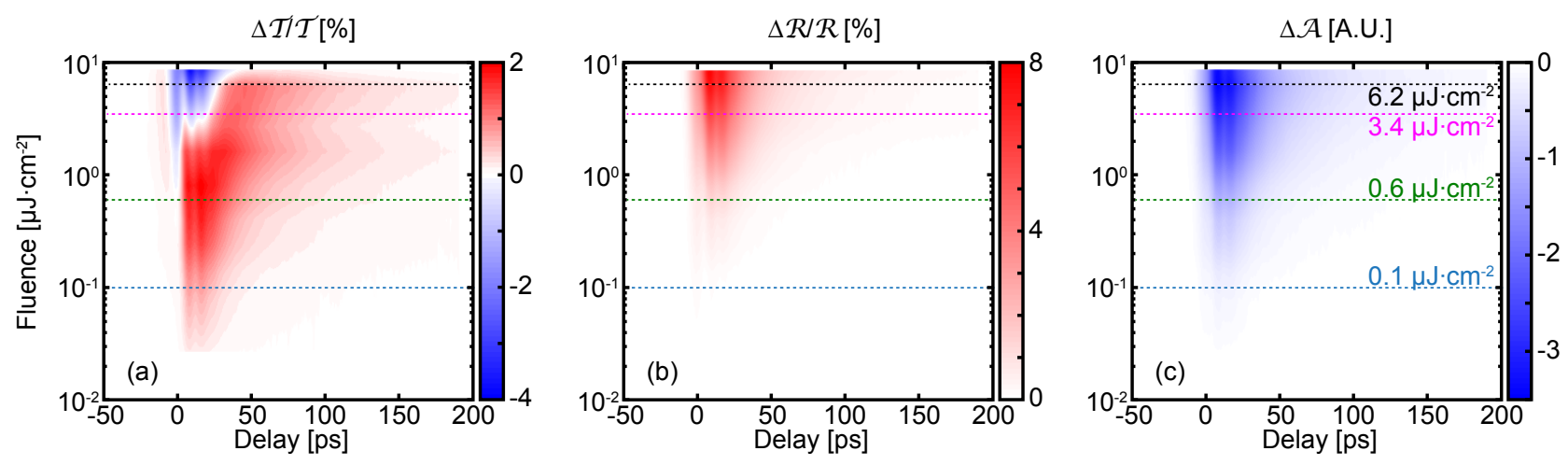

FIG. 2. Measured (a) relative change in transmission, and (b) reflection as a function of pump fluence and pump-probe delay. Change in absorption (c) is calculated from the transmission and reflection data. The dashed lines indicate slices of the data at fluences that produce distinct curves in the relative transmission data. 


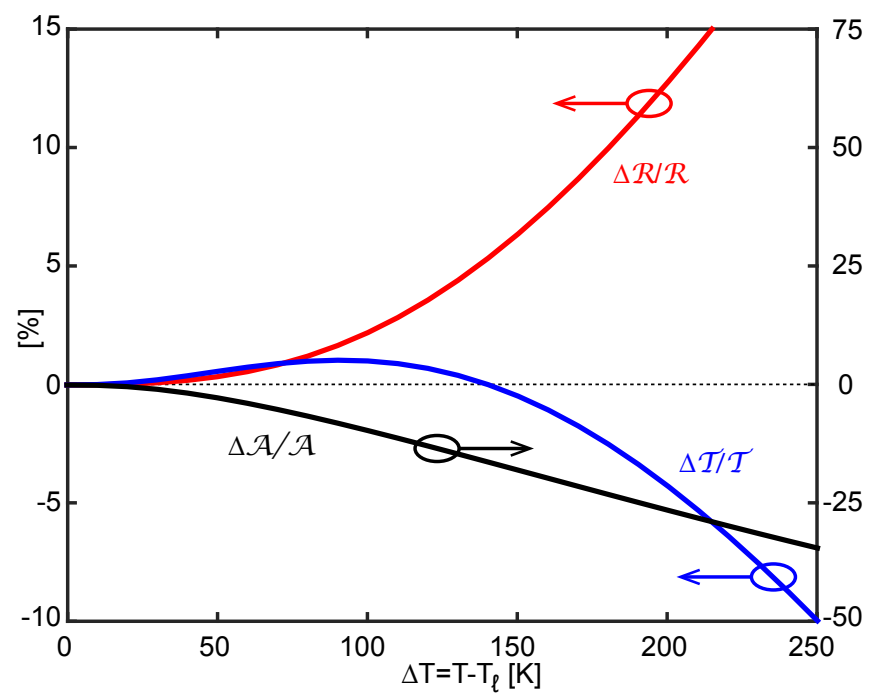

FIG. 3. Calculated optical parameters for multilayer epitaxial graphene on silicon carbide substrate as a function of change in temperature. Parameters for calculation are for 50 graphene layers with $n=1.65 \times 10^{10} \mathrm{~cm}^{-2}, \tau_{s}=300 \mathrm{fs}$ at a temperature of $T_{\ell}=10 \mathrm{~K}$. 


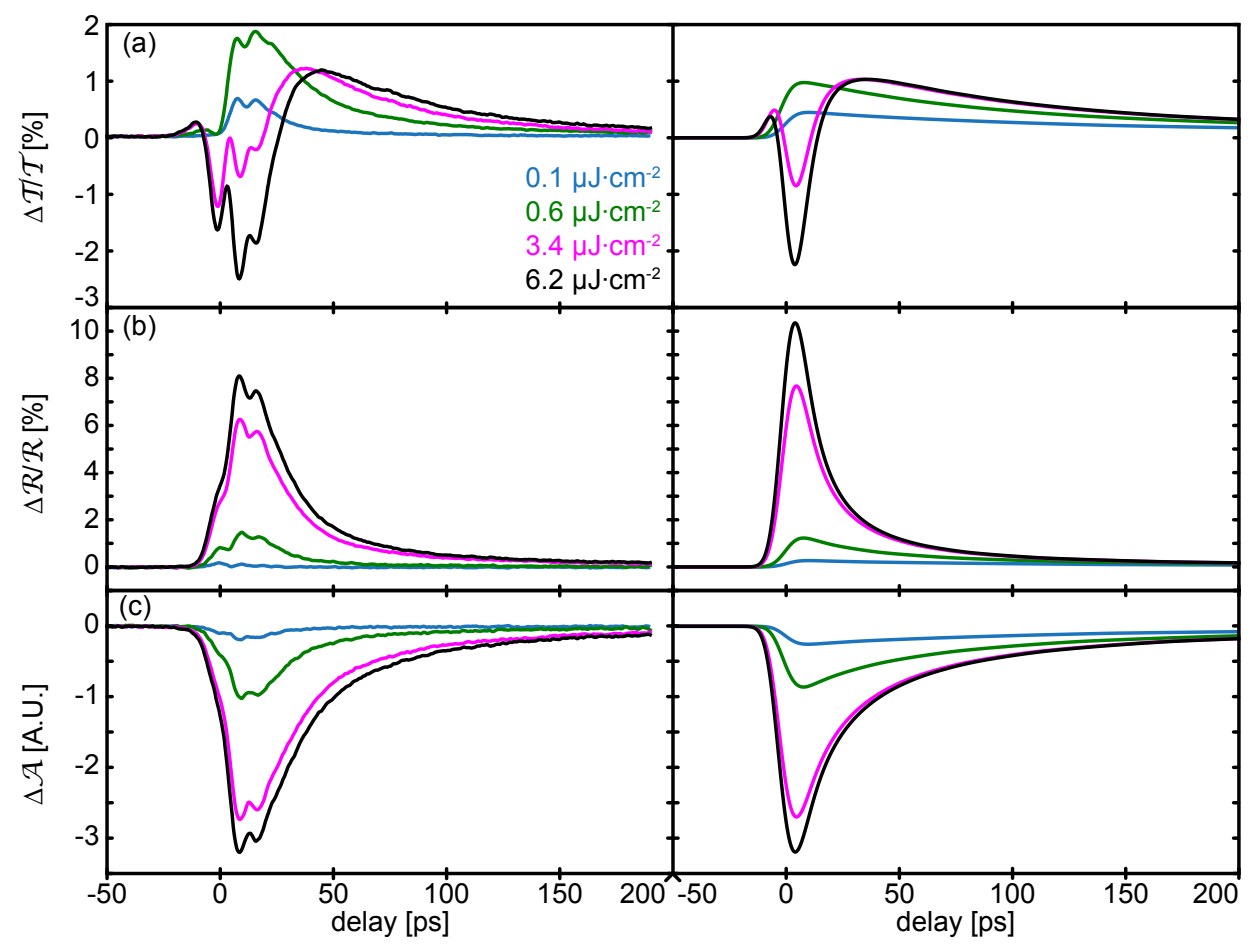

FIG. 4. Measured (left panel) and modeled (right panel) transient pump-probe curves for the four pump fluences defined in Fig. 2. 

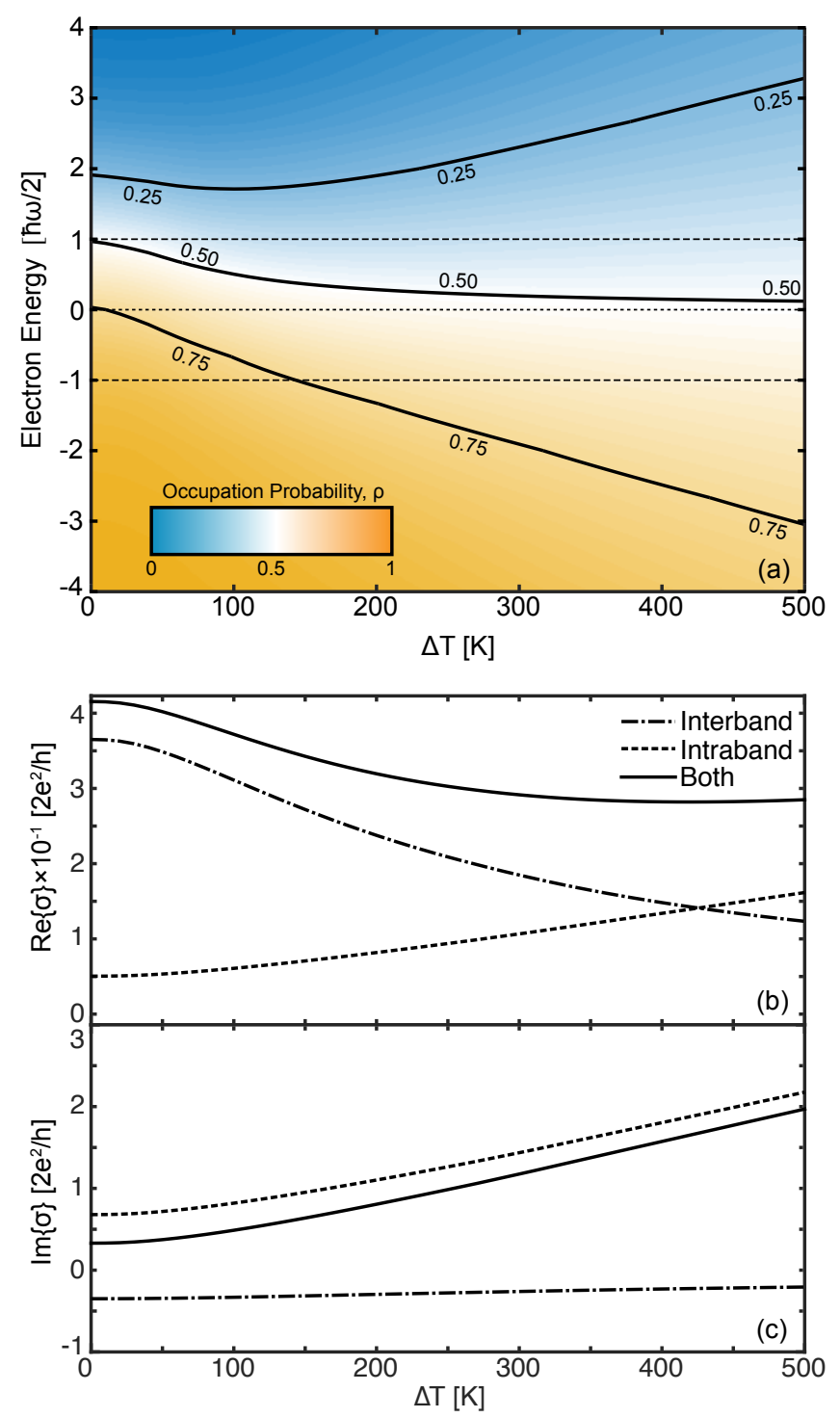

FIG. 5. (a) Contour plot of the occupation probability for different changes in temperature. The ordinate axis is scaled by the photon half energy $(\hbar \omega / 2=15 \mathrm{meV})$; horizontal dashed lines at -1 , 0 , and 1 are plotted for visual reference. Real (b) and imaginary (c) conductivity curves showing intra- and interband contributions versus change in temperature for the photon energy used in the experiment $(\hbar \omega=30 \mathrm{meV})$. 


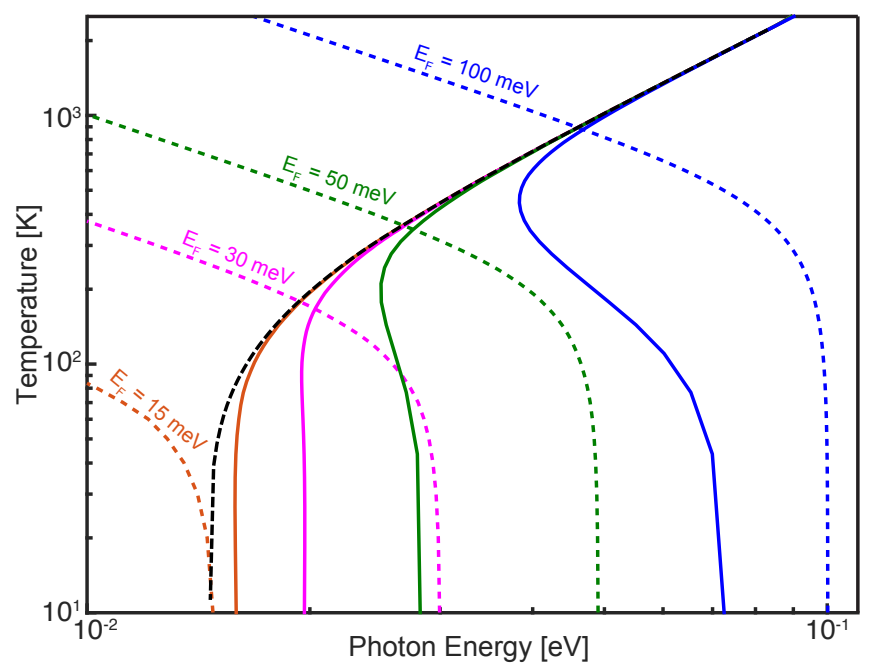

FIG. 6. Plot of temperature where the real part of the conductivity has equal contributions from intra- and interband processes as a function of photon energy for various sample doping levels (solid colored lines) and a charge puddle disorder strength of approximately $20 \mathrm{meV}$. The dashed colored lines show the corresponding temperature dependent chemical potential while the black dashed line indicates a numeric high temperature solution that is independent of doping level. 